\title{
Statistical Inference Method of User Preference on Broadcasting Content
}

\author{
Sanggil Kang ${ }^{1}$, Jeongyeon $\mathrm{Lim}^{2}$, and Munchurl Kim ${ }^{2}$ \\ ${ }^{1}$ Department of Computer Science, College of Information Engineering, \\ The University of Suwon, Hwaseong, Gyeonggi-do, Korea \\ sgkang@suwon. ac. kr \\ ${ }^{2}$ Laboratory for Multimedia Computing, Communications and Broadcasting, \\ Information and Communications University, Daejeon, Korea \\ \{jylim,mkim\}@icu.ac.kr
}

\begin{abstract}
This paper proposes a novel approach for estimating the statistical multimedia user preference by providing weights to multimedia contents with respective to their consumed time. The optimal weights can be obtained by training the statistical system in the sense that the mutual information between old preference and current preference is maximized. The weighting scheme can be done by partitioning a user's consumption history data into smaller sets in a time axis. With developing a mathematical derivation of our learning method, experiments were implemented for predicting the TV genre preference using 2,000 TV viewers' watching history and showed that the performance of our method is better than that of the typical method.
\end{abstract}

\section{Introduction}

With the flood of multimedia content over the digital TV channels, the internet, and etc., users sometimes have a difficulty in finding their preferred content, spend heavy surfing time to find them, and are even very likely to miss them while searching. By predicting or recommending the user's preferred content, based on her/his usage history in content consumptions, the problems can be solved to some extent.

Various preference recommendation techniques can be classified into three possible categories such as the rule-based, collaborative filtering, and inference method. The rule-based recommendation is usually implemented by a predetermined rule, for instance, if -then rule. Kim et al. [1] proposed a marketing rule extraction technique for personalized recommendation on internet storefronts using tree induction method [2]. As one of representative rule-based techniques, Aggrawall et al. $[3,4]$ proposed a method to identify frequent item sets from the estimated frequency distribution using association-rule mining algorithm [5]. Collaborative filtering (CF) technique recommends a target user the preferred content of the group whose content consumption mind is similar to that of the user. Because of the nature of the technique, $\mathrm{CF}$ has been attractive for predicting various preference problems such as net-news [6, 7], e-commerce [8, 9], digital libraries [10, 11], digital TV [12, 13]. In general, rule-based and CF techniques need expensive effort, time, and cost to collect 
a large number of users' consumption behavior due to the nature of their methodologies. However, inference is the technique that a user's content consumption behavior is predicted based on the history of personal content consumption behaviors. Ciaramita et al. [14] presented a Bayesian network [15], the graphical representation of probabilistic relationship among variables which are encoded as nodes in the network, for verb selectional preference by combining the statistical and knowledgebased approaches. The architecture of the Bayesian network was determined by the lexical hierarch of Wordnet [16]. Lee [17] designed an interface agent to predict a user's resource usage in the UNIX domain by the probabilistic estimation of behavioral patterns from the user behavior history.

From the literatures mentioned above, there is one thing not to be overlooked, which is that all data are equally weighted in computing the statistical preference. In this case, recently collected data may not be appreciated because the size of the user's usage history data usually dominates over that of the new data. In general, the recent usage history will give more impact on predicting the future preference than old one. In order to take into the consideration, we provide weights to data with respect to their collected or consumed time.

The objective of our work is to find the optimal weights bringing better performance than the typical methods. In this paper, a new adaptive learning method is proposed for obtaining the optimal weights. Our method executes by partitioning the usage history data into smaller sets in a time axis, on which the weights are provided. Thus, the weighted data can differently reflect their significance on predicting the preference. We utilize a supervised learning technique commonly used in neural network for estimating the weights using the mutual information, which is an optimality index to be maximized during the learning process. Also, the weights are updated whenever a predetermined amount of data is collected.

The remainder of this paper is organized as follows. Section 2 describes a window weighing scheme. Section 3 describes our learning method. In Section 4, we show the experimental results performed on a realistic set of data. We then conclude our paper in Section 5.

\section{Window Weights}

For predicting users' preference, the time that content is consumed can be a critical factor. In order to consider this, we partition the usage history data stored in a chronicle into smaller sets in a time axis and give weights to the frequencies of content in the partitioned datasets. The smaller dataset, named as window in our paper, resides within a predetermined non-overlapped time interval $L$. Usually, the content in the recent windows will give a potent influence on predicting the future preference. In case the data size in those windows is not big, it can not be enough to represent the statistical future preference, which makes us reluctant to use only the latest window. To compensate this problem, we group the windows into two regions: old preference region (OPR) and current preference region (CPR). CPR includes the latest window and is used as a reference for predicting future preference. The OPR has enough windows in order for the estimated conditional probabilities in the region to be able to represent the future preference (FP), compared to CPR. The frequencies 
of content can be modified by assigning weights to the windows in OPR. A set of the window weights can be denoted as $\underline{w}=\left[\begin{array}{llll}w_{1} & w_{2} & \ldots & w_{M}\end{array}\right]$, here $M$ is the total number of windows in the OPR. The statistical preference of the $i^{\text {th }}$ content $x_{i}$ in OPR can be expressed as

$$
\hat{\theta}_{x_{i}}=p\left(X=x_{i} \mid E\right)=\sum_{m=1}^{M} w_{m} n_{i, m} / \sum_{m=1}^{M} w_{m} N_{m}
$$

where $X$ is a set of consumed content, and $N_{m}$ and $n_{i, m}$ is the sample number of whole content and content $x_{i}$ within the $m^{\text {th }}$ window in OPR, respectively. Also, $\hat{\theta}_{x_{i}}$ is the estimated statistical preference of $x_{i}$ for FP with given evidence $E$. From Equation (1), we can see that the conditional probability is a function of the window weights, which means the accuracy of the preference prediction depends upon the values of the weights. As addressed, the latest window can give a big impact on predicting the future preference (FP) so the weights are adjusted in the sense that a set of the content in the OPR, denoted as $\tilde{X}_{O}$, is getting correlated with a set of weighted content in the CPR, denoted as $X_{C}$. Here, $\tilde{X}_{O}$ is the weighted version of $X_{O}$ which is a set of content in the OPR.

In some typical methods, the statistical preference is computed using the entire history dataset, which causes the new coming content not be appreciated in estimating the preference because the size of the user's usage history data usually dominates over that of the new content.. In order to complement this weak point, we rule out the most outdated window when new data is filed up during the next time interval $L$ from the last window in the usage history data. It can be operated by shifting (or sliding) a window at a time and continuing the same processing to find the optimal weights and so on. This scheme can allow an on-line prediction. From the following section, $s$ with the parenthesis indicate the $s^{\text {th }}$ window shifting process.

\section{Determination of Optimal Window Weights}

Our learning method is to determine an optimal set of the window weights in the sense that the mutual information, denoted as $I\left(\tilde{X}_{O}(s) ; X_{C}(s)\right)$, between $X_{C}(s)$ and $\tilde{X}_{O}(s)$ is maximized at the $s^{\text {th }}$ shift. At each window shifting, the weight updates are done based on a gradient ascent algorithm. The weight update continues until the mutual information (MI) reaches the maximum value. The mathematical derivation of our learning method starts with the definition of the MI such as

$$
\begin{aligned}
I\left(\tilde{X}_{O}(s) ; X_{C}(s)\right) & =\log \left(p\left(X_{C}(s) \mid \tilde{X}_{O}(s)\right) / p\left(X_{C}(s)\right)\right) \\
& =\log \left(p\left(X_{C}(s) \mid \tilde{X}_{O}(s)\right)\right)-\log \left(p\left(X_{C}(s)\right)\right) .
\end{aligned}
$$

From Equation (2), the larger the value of the MI, the more $X_{C}(s)$ is correlated with $\tilde{X}_{O}(s)$. We assume that $X_{C}(s)$ includes at least a content to exclude the extreme 


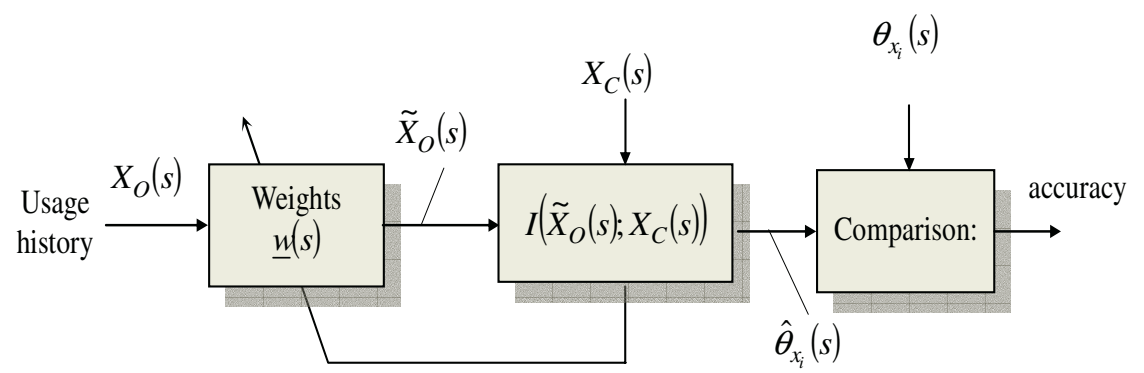

Fig. 1. The schematic of learning method

case in which the equation can not be appropriately applied. For instance, if $X_{C}(s)=\Theta$ then the $\log \left(p\left(\Theta \mid \tilde{X}_{O}(s)\right)\right)$ can not be calculated, where $\Theta$ means the empty set. As searching the optimal weights in the weight space, the partial derivative of the mutual information with respective to the weight $w_{m}(s)$ is calculated as following:

$$
\begin{aligned}
& \partial I\left(\tilde{X}_{O}(s) ; X_{C}(s)\right) / \partial w_{m}(s) \\
& =\partial \log \left(p\left(X_{C}(s) \mid \tilde{X}_{O}(s)\right)\right) / \partial w_{m}(s)-\partial \log \left(p\left(X_{C}(s)\right)\right) / \partial w_{m}(s)
\end{aligned}
$$

where, the MI is composed of the logarithmic functions so it is differentiable at a concerning point in the weight space. Thus, it is feasible to use the gradients. Since the prior probability $p\left(X_{C}(s)\right)$ on the right hand side is not a function of $\underline{w}(s)$, Equation (3) can be simplified as

$$
\partial I\left(\tilde{X}_{O}(s) ; X_{C}(s)\right) / \partial w_{m}(s)=\partial \log \left(p\left(X_{C}(s) \mid \tilde{X}_{O}(s)\right)\right) / \partial w_{m}(s)
$$

In order to compute the partial derivative, we need to formulize $p\left(X_{C}(s) \mid \tilde{X}_{O}(s)\right)$

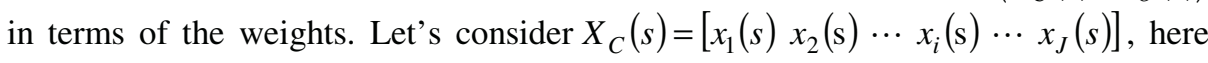
$J$ is the number of attributes in $X_{C}(s)$ and assume that all elements in $X_{C}(s)$ are contained in $\tilde{X}_{O}(s)$. In order to obtain $p\left(X_{C}(s) \mid \tilde{X}_{O}(s)\right)$, we first need to determine the conditional probability $p\left(x_{i}(s) \mid \tilde{X}_{O}(s)\right)$ which means the probability of occurring attribute $x_{i}$ given $\tilde{X}_{O}(s)$. The conditional probability $p\left(x_{i}(s) \mid \tilde{X}_{O}(s)\right)$ is given by

$$
p\left(x_{i}(s) \mid \tilde{X}_{O}(s)\right)=\sum_{m} w_{m}(s) n_{i, m}(s) / \sum_{m} w_{m}(s) N_{m}(s)
$$

The conditional probability $p\left(X_{C}(s) \mid \tilde{X}_{O}(s)\right)$ can be obtained by multiplications of the conditional probabilities of all content in $X_{C}(s)$ under the assumption that the consumption of the contents is independent among them. 


$$
\begin{aligned}
p\left(X_{C}(s) \mid \tilde{X}_{O}(s)\right) & =\prod_{i} p\left(x_{i}(s) \mid \tilde{X}_{O}(s)\right)^{n_{x_{i}}(s)} \\
& =\prod_{i}\left(\sum_{m} w_{m}(s) n_{i, m}(s) / \sum_{m} w_{m}(s) N_{m}(s)\right)^{n_{x_{i}(s)}}
\end{aligned}
$$

where $n_{x_{i}(s)}$ is the number of $x_{i}$ in $X_{C}(s)$. Thus, $\log \left(p\left(X_{C}(s) \mid \tilde{X}_{O}(s)\right)\right)$ in (2) is

$$
\begin{aligned}
\log \left(p\left(X_{C}(s) \mid \tilde{X}_{O}(s)\right)\right) & =\log \left(\prod_{i}\left(\sum_{m} w_{m}(s) n_{i, m}(s) / \sum_{m} w_{m}(s) N_{m}(s)\right)^{n_{x_{i}}(s)}\right) \\
& =\sum_{i} n_{x_{i}}(s)\left(\log \left(\sum_{m} w_{m}(s) n_{i, m}(s)\right)-\log \left(\sum_{m} w_{m}(s) N_{m}(s)\right)\right)
\end{aligned}
$$

Therefore,

$$
\begin{aligned}
& \partial I\left(\tilde{X}_{O}(s) ; X_{C}(s)\right) / \partial w_{m}(s) \\
& =\sum_{i} n_{x_{i}}(s)\left(n_{i, m}(s) / \sum_{m} w_{m}(s) n_{i, m}(s)-N_{m}(s) / \sum_{m} w_{m}(s) N_{m}(s)\right)
\end{aligned}
$$

The weights are updated every epoch defined as one sweep for all data included in the current windows. The amount of update $\Delta w_{m}(s)$ can be obtained using the delta rule such as

$$
\Delta w_{m}(e, s)=\eta \cdot \partial I\left(\tilde{X}_{O}(s) ; X_{C}(s)\right) / \partial w_{m}(s)
$$

where notation $e$ is the number of epochs and $\eta$ is the learning rate which determines the degree of searching step in the weight space during the learning process. We can express the weight update in every epoch $e$ as

$$
w_{m}(e, s) \leftarrow w_{m}(e-1, s)+\Delta w_{m}(e, s) .
$$

However, the point we should not overlook is that the weights may trace to the negative weight space during the learning process according to the initial location of the weights. The negative weights cause a problematic situation in calculating the conditional probabilities which should always be positive. Thus, the weight searching can be caught in a vicious circle as the epoch runs. To avoid this situation, we put restrictions on weight update in order to force weights in only positive space during training. If a weight move to the negative space, we do not update the weight, as seen in Equation (11).

$$
\left\{\begin{array}{lr}
w_{m}(e, s) \leftarrow w_{m}(e-1, s)+\Delta w_{m}(e, s), & \text { if } w_{m}(e, s)>0 \\
w_{m}(e, s) \leftarrow w_{m}(e-1, s), & \text { otherwise }
\end{array}\right\}
$$

Now, we have a question about stopping criteria like "When do we have to stop learning?" In our method, the learning process continues until the predetermined number of epochs is reached, or until the maximum MI is reached. In the case that MI 
reaches the saturation much faster than the predetermined epoch, we pay the overtraining time and effort for the large epoch. To avoid this situation, we stop the training when the MI does not increase any more for some predetermined epoch.

\section{Experimental Results and Analysis}

In this section, we show the numerical accuracy of our learning method by comparing with that of the typical method, that is, no-training method in which no weights are assigned. We applied our method to the Digital TV genre recommendation problem.

For the training and test data set, we used a large set of TV watching history data collected from December 1, 2002 to May 31, 2003, which is provided by AC Nielsen Korea, one of the authorized market research company in Korea. For 2,000 TV viewers, the TV watching history was collected by a set-top box installed in their houses, which can record login and logout time, broadcasting time and day, watched program genre, etc. From the data, we can extract only two evidences such as TV watching time and day for computing the statistical preference of each genre. The genre includes eight attributes such as Education, Drama \& Movie, News, Sports, Children, Entertainment, Information, Others. The considered watching time is only from 6 p.m. to 12 p.m. in our experiment because the user barely watched TV during other time periods. The watching time period was slotted by every two hours for each day. Thus, the set of evidence can be expressed as $E=\{(6$ p.m. $\sim 8$ p.m., Monday), (6 p.m. $~ 8$ p.m., Monday $), \ldots$, (10 p.m. 12 p.m., Sunday $)\}$ with 21 elements. For each case in $E$, we first extracted and arranged the genres of the watched TV programs from each viewer's watching history and then partitioned them by every one week. If there is any missing week, the window of the week was replaced with the next week in order to render training to be possible.

The accuracy is evaluated in terms of the error between the estimated statistical preference and the true preference for the future preference with $\eta=0.1$ and the initial weight vector whose elements are all ones.

$$
\operatorname{Error}(s)=\sum_{i=1}^{J}\left|\hat{\theta}_{x_{i}}(s)-\theta_{x_{i}}(s)\right|
$$

where $\hat{\theta}_{x_{i}}(s)$ and $\theta_{x_{i}}(s)$ is the estimated statistical preference and true preference of $x_{i}$ for the future preference at shift $s$, respectively. As sliding one window at a time until approaching to the last window, we repeated the processes of training and calculating the errors by varying $M$, the number of windows in the OPR, for 2,000 TV viewers. By gender and age group of the viewers, we tabulated the mean error over the viewers, for the typical and our method as shown in the table.

From the table, it is shown that the performances of our method were better than those of the typical method for $10 \mathrm{~s}, 20 \mathrm{~s}, 30 \mathrm{~s}$, and $40 \mathrm{~s}$. For $10 \mathrm{~s}$, around $50 \%$ at maximum improvement was made, for $20 \mathrm{~s}$ and 30 s, around $37 \%$, for 40 s, around $12 \%$. However, for $50 \mathrm{~s}$, it can be stated that there was no improvement. It can be induced that the trend of the preference of 40 s and 50s viewers usually is steadier than 
$10 \mathrm{~s}, 20 \mathrm{~s}$, and 30s. It is hard to provide a unique number of windows in OPR for obtaining optimal performance for all age and gender identity, for instance, the number of window 3 or 4 for 10 s, 4 or 5 for 20 s, etc.

Table 1. The mean errors of the typical method and our method by varying the value of $M$

\begin{tabular}{|l|l|l|l|l|l|l|l|}
\hline \multirow{2}{*}{ Age (Gender) } & Method & \multicolumn{6}{l}{ The number of windows in the OPR, $M$} \\
\cline { 3 - 8 } & & 3 & 4 & 5 & 6 & 7 & 8 \\
\hline \multirow{3}{*}{ 10s (male) } & Typical & 0.24 & 0.21 & 0.22 & 0.2 & 0.24 & 0.23 \\
\cline { 2 - 8 } & Our & 0.12 & $\mathbf{0 . 1}$ & 0.13 & 0.14 & 0.16 & 0.15 \\
\hline \multirow{3}{*}{ 10s (female) } & Typical & 0.22 & 0.2 & 0.21 & 0.24 & 0.23 & 0.25 \\
\cline { 2 - 8 } & Our & $\mathbf{0 . 1 3}$ & 0.15 & 0.16 & 0.15 & 0.14 & 0.15 \\
\hline \multirow{3}{*}{ 20s \& (male) } & Typical & 0.22 & 0.21 & 0.19 & 0.21 & 0.2 & 0.22 \\
\cline { 2 - 8 } & Our & 0.14 & 0.15 & $\mathbf{0 . 1 2}$ & 0.14 & 0.16 & 0.17 \\
\hline \multirow{3}{*}{ 30s \& female } & Typical & 0.2 & 0.19 & 0.22 & 0.19 & 0.21 & 0.22 \\
\cline { 2 - 8 } & Our & 0.14 & $\mathbf{0 . 1 1}$ & 0.13 & 0.15 & 0.13 & 0.12 \\
\hline 30s \&(female) & Typical & 0.22 & 0.23 & 0.2 & 0.22 & 0.22 & 0.23 \\
\cline { 2 - 8 } & Our & 0.13 & 0.14 & $\mathbf{0 . 1 2}$ & 0.13 & 0.14 & 0.13 \\
\cline { 2 - 8 } & Typical & 0.21 & 0.19 & 0.2 & 0.19 & 0.22 & 0.22 \\
\hline & Our & 0.14 & 0.15 & 0.11 & $\mathbf{0 . 1 1}$ & 0.15 & 0.13 \\
\hline 40s \& (male) & Typical & 0.23 & 0.22 & 0.23 & 0.21 & 0.19 & 0.22 \\
\cline { 2 - 8 } & Our & $\mathbf{0 . 1 6}$ & 0.17 & 0.17 & 0.17 & $\mathbf{0 . 1 6}$ & 0.18 \\
\hline 40s\& (female) & Typical & 0.19 & 0.18 & 0.19 & 0.18 & 0.2 & 0.19 \\
\cline { 2 - 8 } & Our & 0.17 & $\mathbf{0 . 1 6}$ & 0.17 & $\mathbf{0 . 1 6}$ & 0.17 & 0.18 \\
\hline 50s \& (male) & Typical & 0.14 & 0.15 & 0.14 & 0.15 & 0.14 & 0.14 \\
\cline { 2 - 8 } & Our & 0.15 & 0.14 & 0.13 & 0.13 & 0.13 & $\mathbf{0 . 1 2}$ \\
\hline 50s\& (female) & Typical & 0.13 & 0.14 & 0.15 & 0.14 & 0.14 & 0.15 \\
\cline { 2 - 8 } & Our & 0.15 & 0.13 & 0.15 & 0.13 & $\mathbf{0 . 1 2}$ & $\mathbf{0 . 1 2}$ \\
\hline
\end{tabular}

\section{Conclusion}

In this paper, we presented a new system for estimating the statistical user preference with introducing the window weights and developing the optimal index, the mutual information, used as a teacher when training the system. By forcing to have old preference be correlated with current preference, the prediction can be good for the near future preference which is correlated with the current preference. From the experimental results, the training speed, which is less than 100 epochs when the initial weighs are all ones, can be acceptable in the practical situation. Also, it was shown that our method was outperformed to the typical method for the $10 \mathrm{~s}, 20 \mathrm{~s}, 30 \mathrm{~s}$, and 40s age.

However, we determined the optimal values of the parameters from the exhaustive empirical experience using 2,000 TV viewers' watching information. The 2,000 viewers might not be enough for the exhaustive experiment. It is needed to collect more viewers' information. Also, we need to do further study for developing an automatic algorithm to estimate the optimal values of parameters for each TV viewer when training our system. 


\section{Acknowledgements}

This research work was carried out at Information and Communications University under the Project titled by "Development of Intelligent Agent and Metadata Management Technology in SmarTV" in 2004 funded by Ministry of Information and Communication in Korean government.

\section{References}

1. J.W. Kim, B.H. Lee, M.J. Shaw, H.L. Chang, M. Nelson, “Application of decision-tree induction techniques to personalized advertisements on internet storefronts," International Journal of Electronic Commerce, vol. 5, no. 3, pp. 45-62, 2001

2. J.R. Quinlan, Induction of decision trees, "Machine Learning," vol. 1, pp. 81-106, 1986

3. R. Aggrawall, T. Imielinski, A. Swami, "Mining association rules between sets of items in large databases," Proc. ACM SIGMOD Int'l Conference on Management of Data, pp. 207216,1994

4. R. Aggrawall, R. Srikant, "Fast algorithms for mining association rules," Proc. $20^{\text {th }}$ Int'l Conference on Very Large Databases, 478-499, 1994

5. M.Z. Ashrafi, D. Tanizr, K. Smith, "ODAM: An optimized distributed association rule mining algorithm," IEEE Distributed Systems Online, vol. 3, no. 3, pp. 1-18, 2004

6. P. Resnick, N. Lacovou, M. Suchak, P. Bergstrom, J. Riedl, "GroupLens: an open architecture for collaborative filtering of netnews," Internet Research Report, MIT Center for Coordination Science, 1994, http://www-sloan.mit.edu/ccs/1994wp.html

7. D.A. Maltz, "Distributing information for collaborative filtering on Usenet net news," $S M$ Thesis, Massachusetts Institute of Technology, Cambridge, MA, 1994

8. J.B. Schafer, J. Konstan, J. Riedl, "Recommender systems in e-commerce," ACM Conference on Electronic Commerce, pp. 158-166, 1999

9. G. Linden, B. Smith, J. York, "Amazon.com recommendations: item-to-item collaborative filtering," IEEE Internet Computing, pp. 76-80, 2003

10. K.D. Bollacker, S. Lawrence, C.L. Giles, "A system for automatic personalized tracking of scientific literature on the web," Proc. ACM Conference on Digital Libraries, pp. 105-113, 1999

11. R. Torres, S.M. McNee, M. Abel, J.A. Konstan, J. Riedl, "Enhancing digital libraries with TechLens+”, ACM/IEEE-CS Joint Conference on Digital Libraries, pp. 228-236, 2004

12. P. Cotter, B. Smyth, "Personalization techniques for the digital TV world," Proc. European Conference on Artificial Intelligence, pp. 701-705, 2000

13. W.P. Lee, T.H. Yang, "Personalizing information appliances: a multi-agent framework for TV programme recommendations," Expert Systems with Applications, vol. 25, no. 3, pp. 331-341, 2003

14. M. Ciaramita, M. Johnson, "Explaining away ambiguity: Learning verb selectional preference with Bayesian networks," Proc. Intl. Conference on Computational Linguistics, pp. 187-193, 2000

15. F. V. Jensen, Bayesian Networks and Decision Graphs, Springer, 2001.

16. G. Miller, R. Beckwith, C. Fellbaum, D. Gross, K.J. Miller, "Wordnet: An on-line lexical database," International Journal of Lexicography, vol. 3, no. 4, pp. 235-312, 1990

17. J.J. Lee, "Case-based plan recognition in computing domains," Proc. The Fifth International Conference on User Modeling, pp. 234-236, 1996 